\title{
Komplexes Fußtrauma
}

$\square$ Thomas Ambacher, Bernd Wittner, Ulrich Holz

\section{Zusammenfassung}

Zur Definition des komplexen Fußtraumas hat sich das 5-Punkte-System nach Zwipp bewährt. Die höchste Inzidenz von komplexen Fußverletzungen weisen polytraumatisierte und mehrfachverletzte Patienten auf. Häufig werden bei diesem Patientengut die Verletzungen des Fußes übersehen oder in ihrer Prognose insbesondere bezüglich des Weichteilschadens falsch eingeschätzt, da zunächst andere initial offensichtliche oder vital bedrohliche Verletzungen im Vordergrund stehen. Die primäre Diagnostik und adäquate chirurgische Versorgung komplexer Fußverletzungen ist von besonderer Bedeutung, da die verzögerte Therapie die Wiederherstellung eines voll gebrauchsfähigen Beines gefährdet. Die Versorgung des komplexen Fußtraumas wird neben den lokalen Verhältnissen entscheidend von der Gesamtverletzungsschwere beeinflusst. Beim Schwerst-Polytraumatisierten ist die primäre Teilamputation indiziert. Sofern die Gesamttraumabelastung einen Erhaltungsversuch erlaubt, entscheidet sich beim komplex traumatisierten Fuß das weitere „Schicksal“ des Fußes bei den geplanten „second“- oder „thirdlook-Eingriffen“. Ein Kompartmentsyndrom des Fußes, welches besonders häufig bei Lisfranc-Luxationsfrakturen auftritt, erfordert stets die notfallmäßige Entlastung, in der Regel durch eine dorsomediane Dermatofasziotomie. Weitere dringliche Versorgungsindikationen stellen zentrale Luxationsfrakturen des Talus und Mittelfußes sowie offene Fersenbeinfrakturen dar. Geschlossene Kalkaneusfrakturen können beim Schwerverletzten sekundär versorgt werden. Bei offenen Frakturen hat sich nach einem Débridement die K-Drahtosteosynthese ggf. in Kombination mit einer Fixateur-externe-Montage bis zur Weichteilkonsolidierung bewährt. Eine temporäre Weichteildeckung ist durch Kunsthaut oder Anlage einer Vakuumversiegelung möglich. Zum definitiven Verschluss des Weichteildefektes kommen je nach Lokalisation neben Sekundär- und dynamischen Nähten, Spalthauttransplantate oder Lappenplastiken infrage. Entscheidend ist ein möglichst intakter Weichteilmantel der Ferse und Fußsohle.

\section{Einleitung}

Das komplexe Fußtrauma tritt häufig bei polytraumatisierten Patienten auf. Typische Unfallmechanismen sind der Sturz aus größerer Höhe sowie der Verkehrsunfall, wobei erhebliche axiale und rotatorische Kräfte auf das Fußskelett einwirken, die zu Zertrümmerungen und Luxationen mit erheblichem Weichteilscha-

OP-JOURNAL 2001; 17: 66-73

(C) Georg Thieme Verlag Stuttgart . New York den führen können [16-18]. Beim Polytraumatisierten stehen in der Regel vital gefährdende und primär offensichtliche Verletzungen im Vordergrund, sodass Fußverletzungen nicht selten initial übersehen werden. Das Übersehen dieser Verletzungen ist besonders tragisch, da beim Überleben des Patienten die langfristige Lebensqualität häufig entscheidend durch die adäquate Versorgung der Fußverletzung bestimmt wird. Nach sekundären Rekonstruktionen und Spätversorgungen kann meist nicht das Ergebnis einer adäquaten Primärversorgung erreicht werden $[6,12,16]$. Daher muss in die erste systematische Ganzkörperuntersuchung im Schockraum der Fuß mit einbezogen werden.

Beim Polytraumatisierten werden Fußverletzungen häufig initial übersehen.

Besonderer Bedeutung kommt beim komplexen Fußtrauma der sofortigen Erfassung eines Kompartmentsyndroms, der korrekten Einschätzung des Weichteilschadens sowie der Diagnostik zentraler Luxationen zu, da Fehlinterpretationen und Spätversorgungen zum Verlust des Fußes führen können $[3,12,18]$. Andererseits kann beim Schwerstverletzten der erzwungene Erhaltungsversuch des komplex traumatisierten Fußes vital gefährdend sein, so dass die initiale Teilamputation in diesen Fällen indiziert ist (Abb.1). Beim Erhaltungsversuch müssen Kompartmentsyndrome und offene Frakturen notfallmäßig im Rahmen der ersten
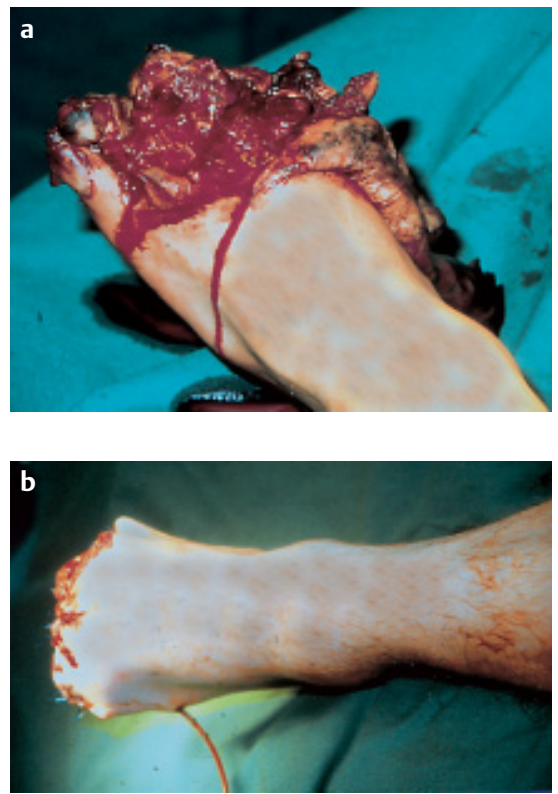

Abb.1 Polytraumatisierter Patient mit komplexer Vorfußtraumatisierung, subtotale Amputation (a). Primäre distale transmetatarsale Amputation (b). 
Operationsphase mitversorgt werden. Eine detaillierte Diagnostik hat ihren obligatorischen Platz nach Stabilisierung und Versorgung vital bedrohlicher Verletzungen [7-9,16-18]. Komplexe Fußverletzungen als Monotrauma sind in der Regel die Folge direkter Kontusionen wie z.B. das Überrollen des Fußes oder Aufprall eines schweren Gewichtes. Beim monoverletzten, ansprechbaren Patienten besteht im Gegensatz zum Polytraumatisierten nicht die Gefahr des Übersehens der Verletzung. In diesen Fällen liegt die besondere Problematik in der korrekten Einschätzung des Weichteilschadens, welcher initial häufig infolge noch vital erscheinender Wundränder als geringgradig eingestuft wird. Da sich das wahre Ausmaß der Kontusionsschäden erst innerhalb von $48 \mathrm{~h}$ ausbildet, ist auch bei banal erscheinender knöcherner Verletzung des Fußskeletts stets eine engmaschige postoperative Verlaufskontrolle, evtl. mit geplantem „secondlook-Eingriff" notwendig $[9,14,18]$.

Das komplette Ausmaß des Weichteilschadens bildet sich verzögert aus, daher ist die engmaschige Verlaufskontrolle obligat.

\section{Definition „Komplexes Fußtrauma“}

Zur Definition des komplexen Fußtraumas hat sich ein 5-Punkte-System gemäß den Kriterien von Zwipp bewährt [17,18]. Dies berücksichtigt zum einen die 5 anatomisch funktionellen Ebenen des Fußes: 1. OSG, 2. Talus, 3. Kalkaneus, 4. Chopart, 5. Lisfranc/Mittelfuß und zum anderen den lokalen Weichteilschaden. Für den Grad des offenen oder geschlossenen Weichteilschadens wird jeweils 1 Punkt vergeben. Bei Überrolltrauma, subtotaler Amputation oder „degloving injury“ 4 Punkte. Ergeben Anzahl der betroffenen Etagen zusammen mit der Punktzahl des Weichteilschadens mindestens 5 Punkte, wird der Fuß als komplex traumatisiert bezeichnet (Abb.2).

\section{Therapieplanung: Amputation oder Rekonstruktion}

Die Versorgung des komplexen Fußtraumas wird neben den lokalen Verhältnissen entscheidend von der Gesamtverletzungsschwere beeinflusst. Trotz der Fortschritte der modernen Intensivmedizin und Wiederherstellungschirurgie welche bei Mono- und Mehrfachverletzten in den meisten Fällen eine erfolgversprechende Fußrekonstruktion auch bei komplexer Traumatisierung erlaubt, können

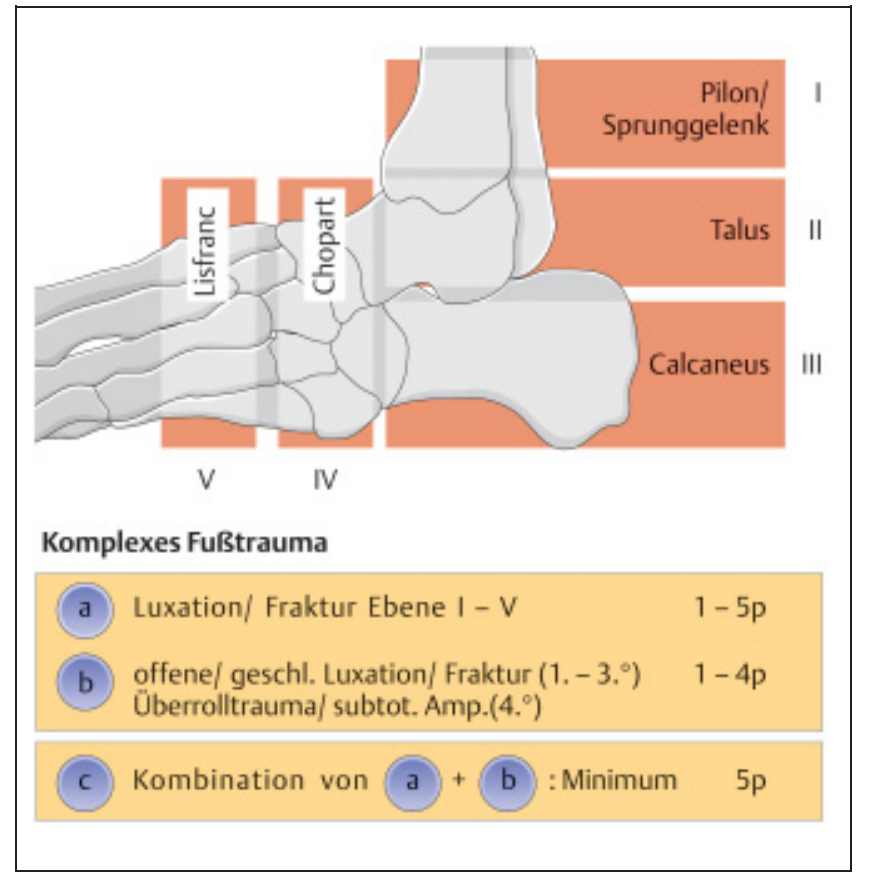

Abb. 2 Die 5 funktionell wichtigen Ebenen des Fußes bei Klassifizierung des komplexen Fußtraumas nach den Kriterien von Zwipp et al. $[17,18]$.

beim schwerstgradig Polytraumatisierten initial aufwendige Erhaltungsversuche den Patienten gefährden. Als Parameter zur Entscheidungshilfe hinsichtlich einer primären Amputation hat sich die Beurteilung des Schweregrades der Gesamtverletzung mittels eines Traumascores etabliert. Tscherne empfiehlt nach den Kriterien des Hannoveraner Polytrauma-Scores (PTS) beim komplexen Fußtrauma und gleichzeitigem Vorliegen eines PTS-Score von 3-4 die primäre Amputation, beim mittelgradigen Polytrauma (PTS 2) die individuelle Entscheidung und bei PTS 1 den Erhaltungsversuch $[7,18]$.

Neben dem Schweregrad der Gesamtverletzung hängt die Entscheidungsfindung hinsichtlich Amputation oder Rekonstruktion von den knöchernen Verletzungen des Fußskeletts, dem Ausmaß des lokalen Weichteilschadens sowie dem Durchblutungs- und neurologischen Status ab. Zur Objektivierung des Schweregrades der Fußverletzung hat sich international der ,mangled extremity severity score" (MESS) als besonders aussagekräftig erwiesen [4]. Ziel dieses Scores ist die Selektion der Extremitätenverletzungen mit schwerem Weichteilschaden, bei denen ein Erhaltungsversuch erfolgversprechend erscheint. Hierzu werden Weichteil-Knochen-Verletzung, periphere Ischämie, Kreislaufverhältnisse und Patientenalter nach einem Punkteschema bewertet. In einer prospektiven Studie an 26 Patienten mit offenen Frakturen der unteren Extremitäten führte ein MESSScore-Wert $>7$ stets zur Amputation.

Beim schwerstgradig Polytraumatisierten können aufwendige Erhaltungsversuche des Fußes den Patienten vital gefährden.

Sofern man sich anhand dieser Kriterien zum Erhaltungsversuch entschließt, sollte innerhalb von $48 \mathrm{~h}$ ein ,secondlook" erfolgen, da sich das Vollbild des Weichteilschadens verzögert ausbildet. Nachdébridements sind häufig erforderlich. Erst im Rahmen dieser Operation teilweise erst durch weitere planmäßige Revisionen kann sicher entschieden werden, ob der Erhalt des Fußes erfolgversprechend und sinnvoll erscheint und das definitive Therapiekonzept bezüglich Teilamputation oder Rekonstruktion festgelegt werden [7-9,16-18].

\section{Amputation}

\section{Festlegen der Amputationshöhe}

Zur Bestimmung der Amputationshöhe ist es notwendig, dass die Durchblutungsverhältnisse unter Verzicht auf eine Blutsperre evaluiert werden. Dies gilt sowohl für den Primäreingriff als auch für die „second-look-Operation“, welche bei grenzwertigen Befunden obligatorisch ist. Sämtliche Areale, die nach Kompartmentspaltung und Reposition der Frakturen und Luxationen keine ausreichende Kapillardurchblutung aufweisen, müssen 


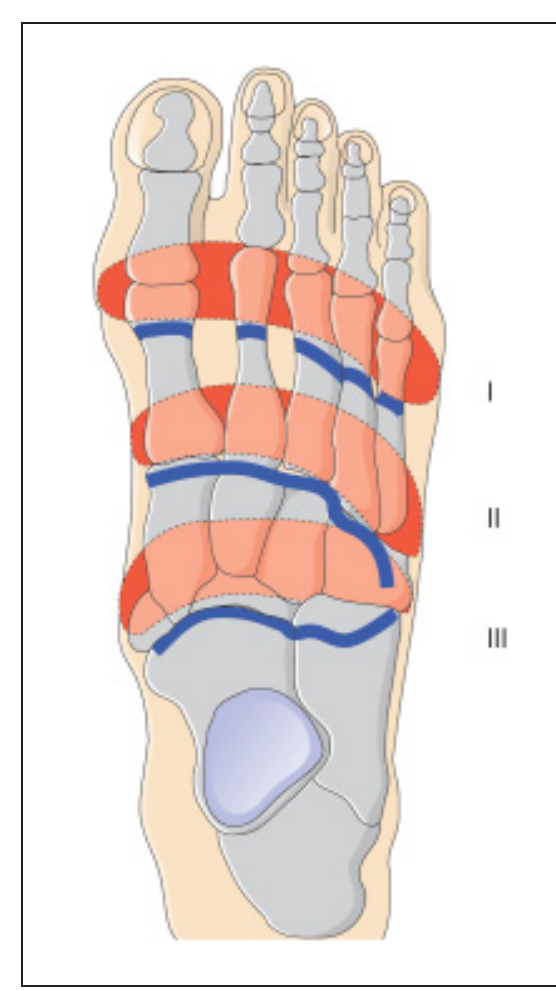

Abb.3 Klassische Amputationslinien am Fuß: I distale transmetatarsale Amputation, II tarsometatarsale Exartikulation Lisfranc, III transtarsale Exartikulation Chopart aus [18].

abgesetzt werden. Die Amputation erfolgt dabei in der Regel in Höhe der 3 klassischen Amputationshöhen: distal transmetatarsal, tarsometatarsal oder transtarsal (Abb.3). Ob die initial gewählte Amputationshöhe ausreichend war, kann frühestens bei der Kontrolle des Stumpfes am 2.-3. postoperativen Tag beurteilt werden. Im Zweifelsfall sind weitere Revisionen mit Nachdébridement oder Nachamputation notwendig $[4,6,14,16-18]$.

\section{Amputationstechniken}

Wie bereits erwähnt, muss zur sicheren Vitalitätsbeurteilung die Operation ohne Blutsperre erfolgen. Nicht nur nekrotisches, sondern auch ernährungsgestörtes und ernährungsgefährdetes Gewebe muss vollständig im Gesunden entfernt werden.

Radikales Débridement mit vollständiger Entfernung von nekrotischem und ernährungsgefährdetem Gewebe.

Als ernährungsgefährdet sind Sehnen, Bänder und Faszien, die keine eigene

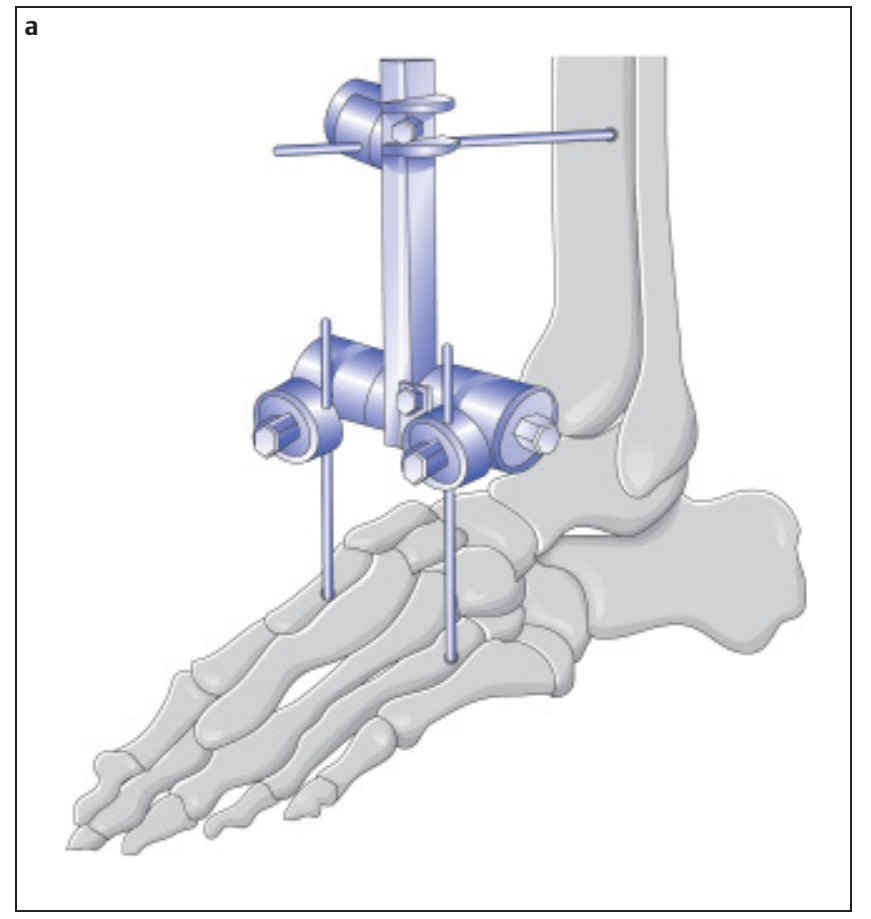

Abb. 4 Tibiotarsale Transfixation schematisch (a) und in der Praxis (b) zur temporären Ruhigstellung und Entlastung der Weichteile.
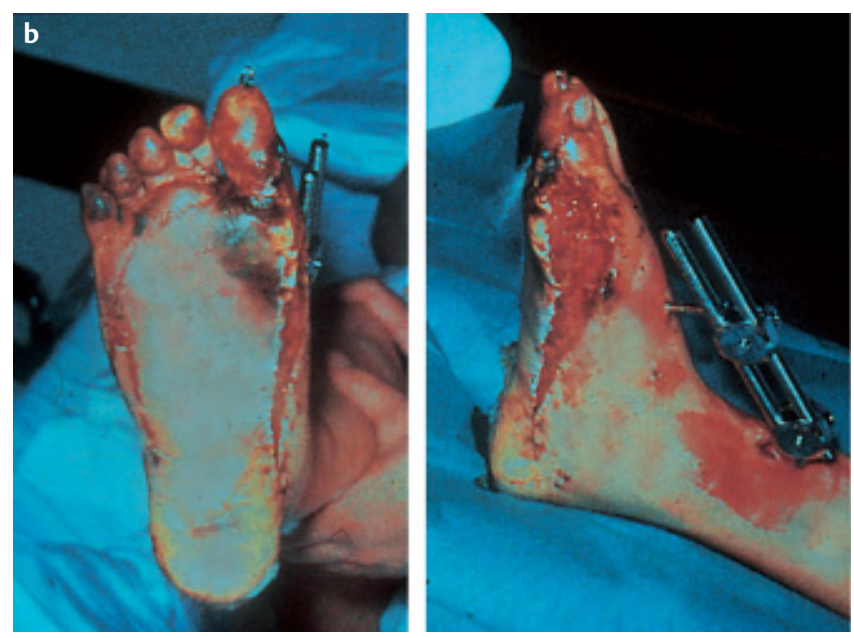

Blutversorgung aufweisen anzusehen und daher sorgfältig zu resezieren. Bei der Exartikulation ist die subchondrale Knochenschicht zu schonen. Die Knochendurchtrennung ist immer im spongiösen Bereich anzustreben. Die sorgfältige Wunddrainage ist unverzichtbar. Frakturen und Luxationen des Stumpfes werden am günstigsten minimal-invasiv durch Kirschner-Drähte reponiert und retiniert. Eine zusätzliche temporäre tibiotarsale Fixateur-externe-Montage kann bis zur Konsolidierung der Weichteile hilfreich sein (Abb.4). Die für eine spätere gute Steh- und Gehfunktion erforderliche Fußstatik und Funktion kann sich teilweise nicht ausschließlich an den klassischen Amputationshöhen ausrichten, sondern muss darüber hinaus in Abhängigkeit vom vorliegenden Weichteildefekt sekundäre Sehnenverlagerungen und aufwendige plastische Verfahren umfassen [6].

Zehenamputation: An der 2.-5.Zehe sollten die Grundphalangen möglichst mit intakter Streckaponeurose erhalten werden. Zur Weichteildeckung des Stumpfes sollte ein plantarer Lappen verwendet werden. An der End- und Mittelphalanx sollte zur Vermeidung von Durchspießungen des Knochenstumpfes und einer Krallenstellung die Exartikulation bevorzugt werden. Im Gegensatz dazu ist an der Großzehe aufgrund ihrer Stützfunktion das Vorgehen von größtmöglicher Substanzerhaltung bestimmt. Die Sesambeine der Großzehe bilden 
einen Teil der Auftrittsfläche und sollten daher nicht exstirpiert werden $[6,17]$.

Mittelfußamputation: Die Amputation auf Mittelfußniveau orientiert sich in der Regel nicht an anatomisch vorgegebenen Linien, sondern wird von der Ausdehnung des Weichteilschadens bestimmt. Da die streckseitige Haut für die Belastung auf Dauer ungeeignet ist, ist hier insbesondere die Durchblutungssituation der plantaren Weichteile, die zur Stumpfdeckung herangezogen werden, entscheidend. Empfohlen wird die basis- oder köpfchennahe Absetzung, da diaphysäre Stümpfe erfahrungsgemäß zu Belastungsproblemen durch Knochenrarefizierung und mögliche Anspießung der Weichteile führen [6-9].

Exartikulation in der Lisfranc-Linie: Amputationen auf Höhe der Lisfranc-Linie sind häufig bei schwerer Quetschverletzung des Vorfußes und des distalen Mittelfußes notwendig. Aufgrund des verwinkelten Verlaufs dieser Gelenklinie sind allerdings in der Regel transossäre Resektionen erforderlich. Die ursprüngliche Gelenklinie kann meist nicht erhalten werden, da für die Absetzung die Weichteilverhältnisse maßgebend sind. Zur Vermeidung einer späteren Spitzfußfehlstellung werden nach Weichteilkonsolidierung die Extensoren in leichter Dorsalflexion des Restfußes durch die Cuneiformia und das Kuboid gezogen und plantar in sich vernäht $[8,9,11]$

Bei der Amputation richtet sich die definitive Absetzungslinie nach den Weichteilverhältnissen.

Exartikulation in der Chopart-Linie: Durch den einseitigen Zug des M. triceps surae gerät der Original-Chopart-Stumpf mit Absetzung in der Chopart-Gelenklinie in eine nicht belastbare Spitz-Klumpfußstellung verbunden mit einer funktionell ungünstigen Beinverlängerung. Dies erfordert sekundäre Korrekturen in Form einer Arthrodese des oberen und unteren Sprunggelenkes. Alleinige Sehnenverlagerungen führen meist nicht $\mathrm{zu}$ einem zufriedenstellenden Ergebnis $[8,11]$.

Transkalkaneare Amputation: Bei der transkalkanearen Amputation nach Pirogoff können alle zerquetschten Weichteile des Fußes radikal entfernt werden, so dass dieses Verfahren beim komplexen Fußtrauma die sicherste Methode zur Vermeidung sekundärer Infektionen darstellt. Die Original-Pirogoff-Amputation

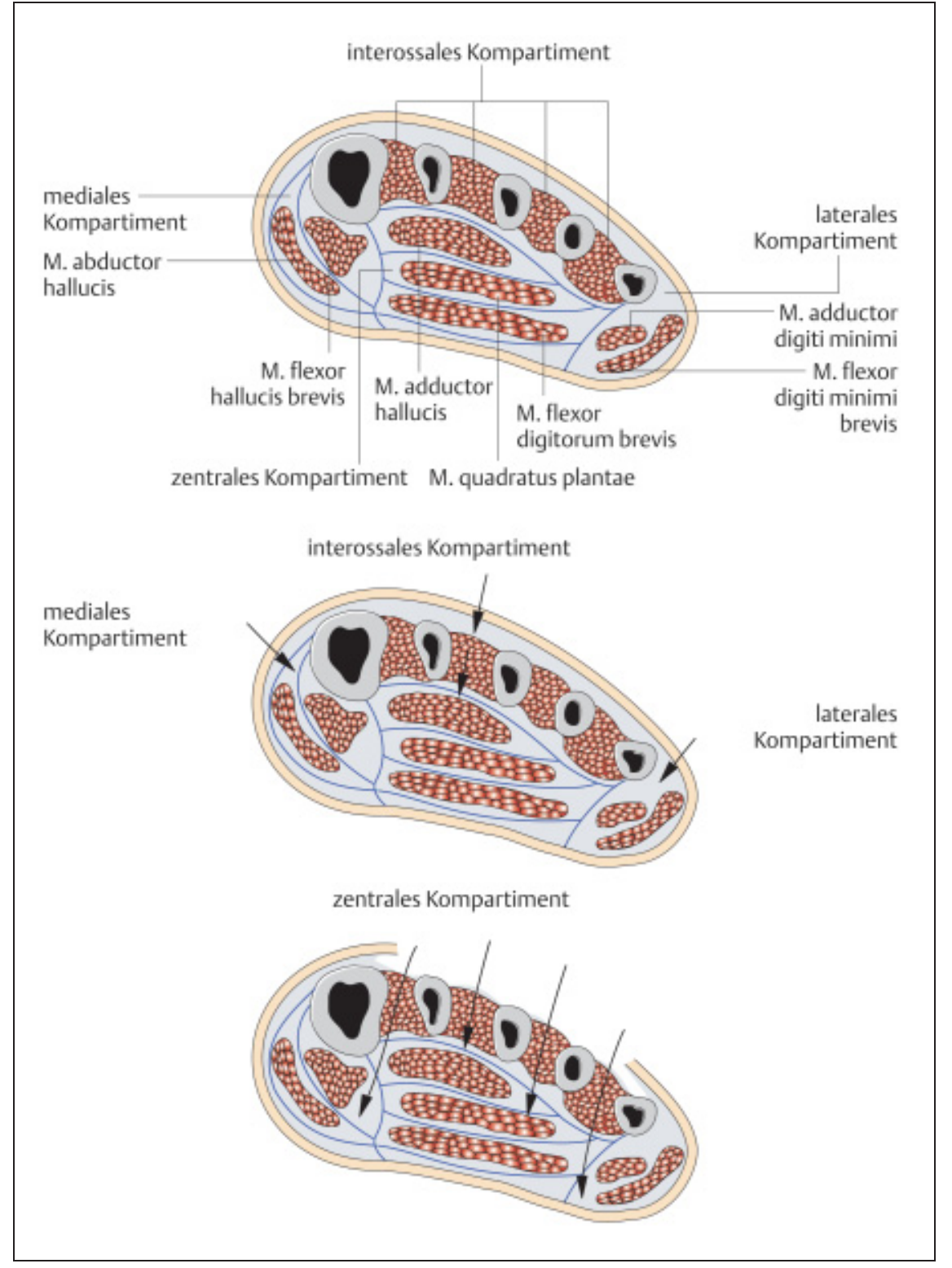

Abb. 5 Schematische Darstellung der Kompartimente des Vor- und Mittelfußes. Über die dorsale Dermatofasziotomie können das zentrale, mediale und laterale Kompartiment entlastet werden.

mit Unterstellung des um $70^{\circ}$ gekippten Tuber calcanei nach Resektion der Knöchelgabel und Exstirpation der Rückfußskelettanteile führt allerdings wegen der druckempfindlichen Fersenhaut zu einem nicht endbelastbaren Stumpf. Daher wurden in den letzten Jahrzehnten modifizierte Verfahren mit Endbelastbarkeit entwickelt. Diese sind immer dann empfehlenswert, wenn einerseits eine weiter peripher gelegene Amputation nicht mehr möglich ist, andererseits aber eine Unterschenkelamputation vermieden werden kann. Allen Modifikationen ist gemeinsam, dass der Kalkaneus nach Resektion der Knöchelgabel dem Unterschenkel so unterstellt wird, dass vollbelastbare Rückfußsohlenhaut in der Belastungszone verbleibt $[17,18]$.

\section{Rekonstruktion}

\section{Kompartmentsyndrom des Fußes}

Wie in allen Muskellogen des Körpers kann sich auch in denjenigen des Fußes ein Kompartmentsyndrom entwickeln. Seine Entstehung in dieser Lokalisation wird durch 3 Faktoren begünstigt:

- Der Fuß, speziell die Fußwurzel und der Mittelfuß, erleiden häufig lokale Quetschverletzungen mit hoher Krafteinwirkung 
- Der „matratzenartige“ Aufbau der Planta pedis mit kräftiger Septierung und dazwischenliegender Muskulatur erlaubt die federnde Aufnahme hoher Belastungen. Dieses Bauprinzip lässt aber den einzelnen Muskellogen sehr wenig Elastizität. So zeigt sich experimentell bei einer Volumenvergrößerung von $6 \mathrm{ml}$ in der Loge des M. flexor digitorum brevis bereits ein Druckanstieg auf $45 \mathrm{~mm} \mathrm{Hg}$

- Die im Endstromgebiet des Fußes bereits häufig reduzierte arterielle Durchblutung setzt die Toleranz gegen Druckerhöhungen in einem Kompartiment noch zusätzlich herab.

An der Planta pedis liegen 3 voneinander isolierte Muskellogen. Im englischen Schrifttum wird die mittlere Loge nochmals unterteilt, so dass hier 4 Logen unterschieden werden. Am Vorfuß finden sich die Kompartimente der Mm. interossei sowie dasjenige des M. abductor hallucis [3,12] (Abb.5).

\section{Klinische Symptomatik}

Kompartmentsyndrome sind eine regelmäßig auftretende Komplikation des komplexen Fußtraumas. Von einigen Autoren wird zur Diagnostik die Druckmessung empfohlen $[3,9,12,18]$. Unseres Erachtens stellt jedoch das Fußkompartment ebenso wie das Kompartmentsyndrom anderer Lokalisierung eine primär klinische Diagnose dar.

Leitsymptome des Kompartmentsyndroms am Fuß ist die massive Weichteilschwellung.

Leitsymptom ist der inspektorische Befund einer massiven Schwellung des Fußes (Abb.6). Häufig fällt darüber hinaus eine deutliche Fehlstellung und Deformierung des Fußes infolge der zugrundeliegenden knöchernen Verletzungen auf. Das Fußkompartment tritt besonders häufig nach Chopart- und Lisfranc-Luxationsfrakturen sowie gelegentlich bei Talus- und Kalkaneusfrakturen auf $[8,11,16,17]$. Beim ansprechbaren Patienten mit komplexem Fußtrauma ist neben der ausgeprägten Weichteilschwellung der nicht adäquat auf Analgetika ansprechende Schmerz ein wichtiges Warnsymptom. Sensibilitätsausfälle stellen bereits ein Spätsymptom dar und sind ein Hinweis für möglicherweise irreversible Folgeschäden. Aufgrund der hohen Inzidenz von Kompartmentsyndromen des Fußes bei mehrfach- und polytraumatisierten Patienten muss daher die ge- zielte Untersuchung der Füße obligatorischer Bestandteil der systematischen Ganzkörperuntersuchung im Schockraum sein, um diese Verletzung nicht zu übersehen. An Spätfolgen sind bei insuffizienter Diagnostik und Therapie Parund Dysästhesien, die Ausbildung von Hammerzehen und/oder einem Hohlfuß sowie Beugekontrakturen der Zehengrundgelenke mit Beeinträchtigung der Abrollbewegung zu erwarten [12].

\section{Operationsindikation und -technik}

Entscheidend für die Indikationsstellung zur Logenspaltung ist der klinische Verdacht auf ein Fußkompartment anhand der ausgeprägten Weichteilschwellung.

Die Indikation zur Kompartmentspaltung sollte aufgrund des klinischen Befundes gestellt werden und nicht anhand gemessener intrakompartimenteller Druckwerte.

Die Kompartmentspaltung erfolgt üblicherweise über eine dorsomediane Dermatofasziotomie mit Durchtrennung der Fascia cruris und dorsalis pedis sowie des distalen Retinaculums und Spaltung aller Kompartimente. Eine zusätzliche laterale Inzision ist meist nicht erforderlich. Bei gleichzeitigem Vorliegen eines Unterschenkelkompartments wird die Fasziotomie nach proximal verlängert und das proximale Retinaculum mit durchtrennt. Die Deckung des entstehenden Weichteildefektes erfolgt zunächst durch

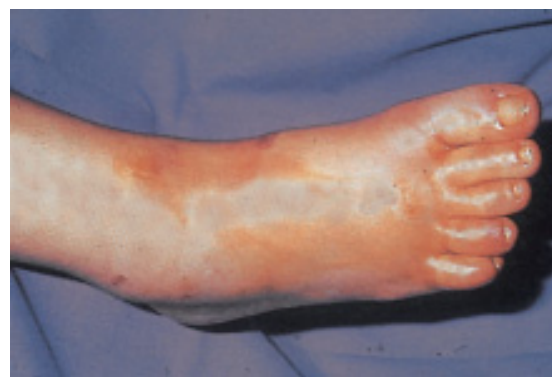

Abb.6 Klinisches Bild eines Kompartmentsyndroms des Fußes. Massive Weichteilschwellung mit gespannter, glänzender Haut.
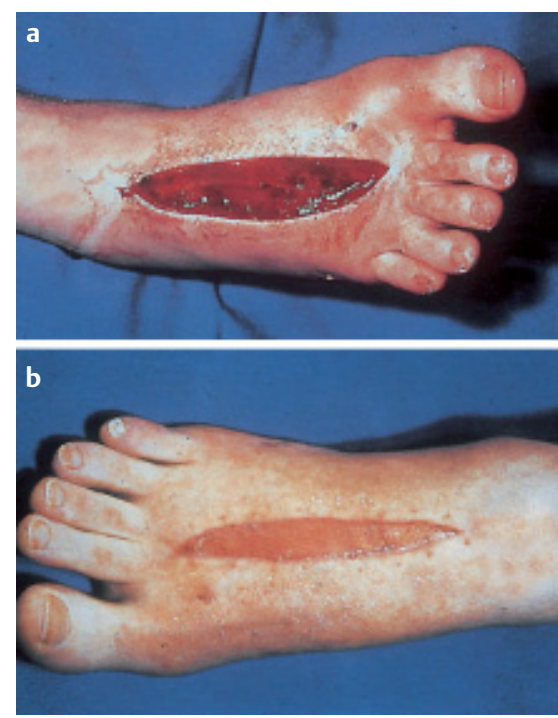

Abb.7 Befund nach dorsaler Dermatofasziotomie beim Fußkompartment-Syndrom (a). Deckung des Weichteildefektes mit Spalthaut (b).

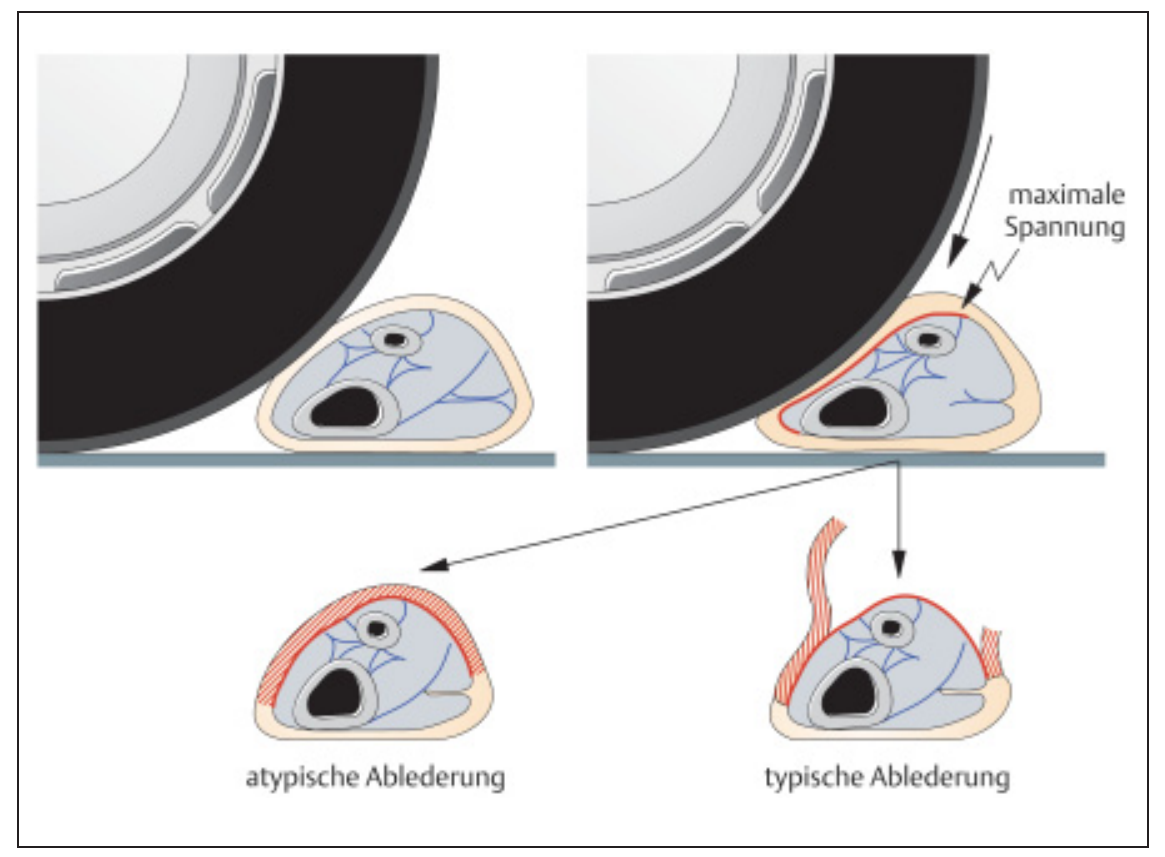

Abb. 8 Decollementverletzung. Typischer Unfallmechanismus beim Überrolltrauma. 

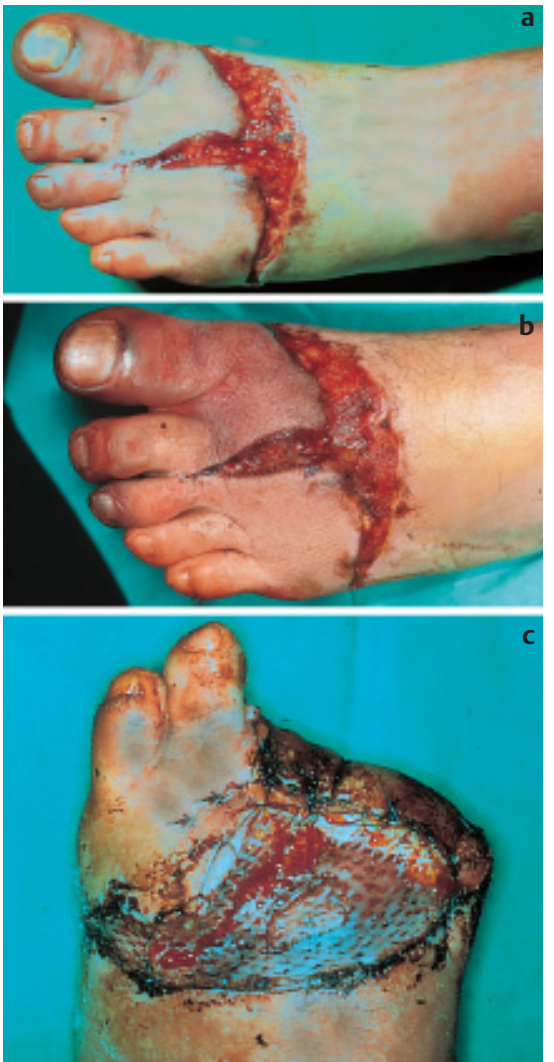

Abb.9 Primärer Befund nach Überrolltrauma des Fußes mit Kontusions-/Decollementverletzung des Vor- und Mittelfußes (a). Beginnende sekundäre Demarkierung nekrotischer Areale (b). Sekundäre Amputation der nekrotischen I. - III. Zehe und Deckung des Weichteildefektes mit Spalthaut (c).

Kunsthaut. Nach Abschwellung ist eine schrittweise Wundrandadaptation mittels dynamischer Nähte oder eine Deckung mittels Spalthaut möglich [3,12,18] (Abb. 7).

\section{Versorgung von Weichteildefekten}

Jeder Hautweichteildefekt stellt für eine benachbarte Fraktur oder Osteosynthese ein ständiges Infektionsrisiko dar. Um eine drohende Wundinfektion im zerstörten Gewebe zu verhindern, ist bei der Erstversorgung ein radikales Débridement erforderlich $[1,15]$.

Im Rahmen der Erstversorgung muss bei Weichteildefekten ein radikales Débridement erfolgen.

Die exakte Beurteilung des Weichteilschadens gestaltet sich vor allem bei Decollementverletzungen häufig schwierig, da sich nekrotische Areale erst sekundär demarkieren (Abb.8) [14,15]. Ein primär
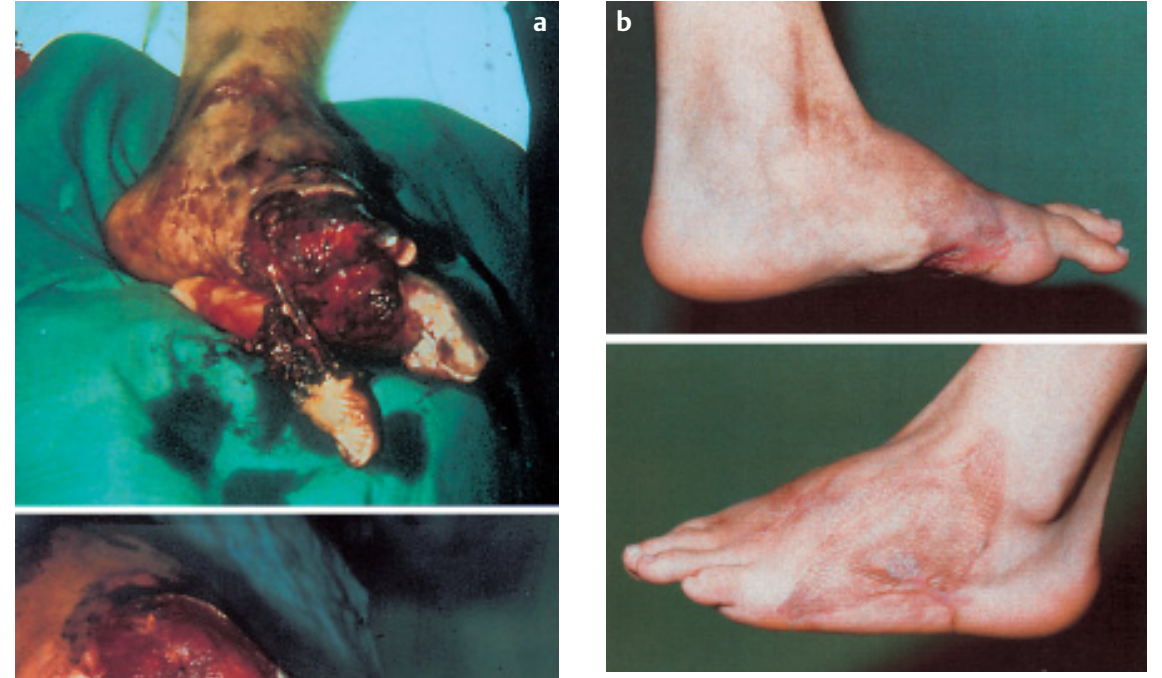

erzwungener Weichteilverschluss ist nicht empfehlenswert, da bei der Erstversorgung eine sichere Abgrenzung zwischen vitalem und geschädigtem Gewebe meist nicht möglich ist. Das Verfahren für den definitiven Defektverschluss sollte daher erst bei einem geplanten second look festgelegt werden. Beim komplexen Fußtrauma ist die konsequente Resektion zerstörten Gewebes von besonderer Bedeutung, da die Durchblutungsverhältnisse infolge vorbestehender systemischer Erkrankungen im Endstromgebiet des Fußes häufig reduziert sind und somit ein erhöhtes Infektionsrisiko sowie ungünstigere Voraussetzungen für eine spontane Erholung bestehen.

Beim schweren Weichteilschaden sollte das endgültige Therapiekonzept im Rahmen eines geplanten secondlook festgelegt werden.

Zur Versorgung von Weichteildefekten stehen verschiedene Maßnahmen zur Verfügung, deren Einsatz von Ausdehnung und Tiefe des Defektes, von der Granulationsfähigkeit des Wundgrunds, von der Lokalisation am Fuß sowie von der Vitalität der an den Defekt angrenzenden Weichteile abhängt [1,2,5,15,17] (Abb.9).

Bei granulationsfähigem Wundgrund am Fußrücken können oberflächliche Defekte nach temporärer Deckung mittels
Abb.10 Komplexes Fußtrauma mit Amputation des I. Strahls (a). Nach Weichteildébridement und Granulation der Defektwunde Deckung mit Spalthaut (b), problemlose Vollbelastung.
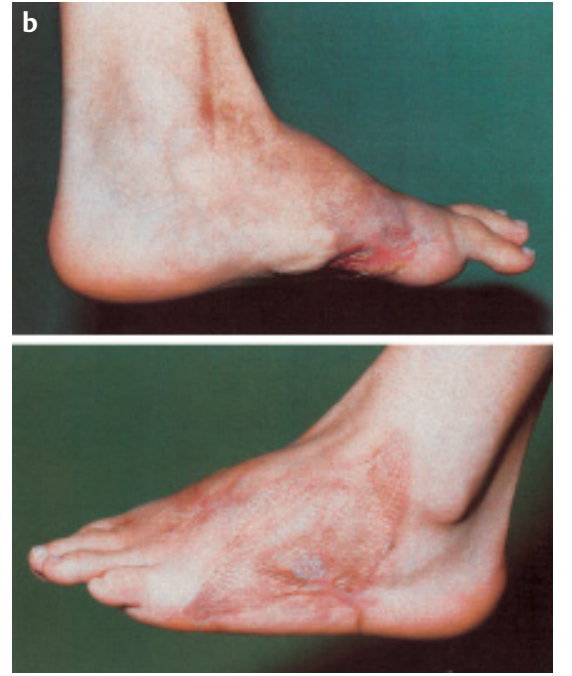

Kunsthaut durch dynamische Nähte sukzessive verschlossen werden [2]. Ist aufgrund der Ausdehnung die spannungsfreie Wundrandadaptation nicht möglich, kommt eine Deckung mit Spalthaut in Frage (Abb.10). Bei tieferen Defekten und unzureichender Granulation des Wundgrundes hat sich zunehmend die Anlage einer Vakuumversiegelung bewährt. Dadurch kann eine Verkleinerung der Fläche und ein Hochgranulieren bis knapp unterhalb des Hautniveaus erreicht werden und der verbliebene Defekt mittels Sekundärnaht oder Spalthaut gedeckt werden $[1,15,18]$. Nicht granulationsfähiger Wundgrund bedarf des Einsatzes von aufwendigen Lappenplastiken welche am Fußrücken allerdings selten erforderlich sind. Zur Erzielung einer belastungsfähigen Weichteilrekonstruktion von Defekten der Fußsohle und der Ferse sind lokale gefäßgestielte Verschiebelappen am besten geeignet, aufgrund des Weichteilschadens jedoch häufig nicht möglich. In diesen Fällen kommt als letzter Ausweg ein freier mikrovaskulärer Muskellappen in Frage, der jedoch mit einer nicht unerheblichen Komplikationsrate behaftet ist [5].

\section{Frakturen im Rahmen des komplexen Fußtraumas}

Talusfrakturen im Rahmen eines komplexen Fußtraumas werden nach denselben 
Prinzipien wie beim Monoverletzten in der Regel notfallmäßig versorgt.

Bei schwerem Weichteilschaden empfiehlt sich die temporäre tibiotarsale Transfixation.

Talusfrakturen im Rahmen eines komplexen Fußtraumas sollten nach den Prinzipien der Monoverletzung notfallmäßig versorgt werden.

Die Therapieplanung der knöchernen Verletzungen sollte anhand einer CTDiagnostik erfolgen. Bei entsprechendem Weichteilschaden wird die Dermatofasziotomie über den anteromedialen $\mathrm{Zu}-$ gang zur Fraktur ausgeführt. Bis zur Konsolidierung der Weichteilverhältnisse empfiehlt sich die tibiotarsale Transfixation. Stellt die Talusfraktur an sich das Komplextrauma dar, hängt die Behandlungsstrategie von den Rekonstruktionsmöglichkeiten im Einzelfall ab. Bei zentralen Frakturen Typ Hawkins III/IV und keiner erfolgversprechenden Rekonstruktion des USG empfiehlt sich die sekundäre Arthrodese, sobald die Weichteilverhältnisse den Eingriff erlauben. Für den umgekehrten Fall eines rekonstruierbaren Subtalargelenkes jedoch nicht wiederherzustellender OSG-Kongruenz sollte die sekundäre OSG-Arthrodese angestrebt werden [16-18].

Geschlossene Kalkaneus- und Mittelfußfrakturen können mit aufgeschobener Dringlichkeit versorgt werden.

Geschlossene Kalkaneusfrakturen mit Weichteilschaden $\mathrm{I}^{\circ}-\mathrm{II}^{\circ}$ können problemlos sekundär innerhalb von 10 Tagen versorgt werden. Im Gegensatz dazu stellt die Weichteilschädigung $\mathrm{III}^{\circ}$ eine dringliche Indikation dar. Häufig kommt beim Mehrfachverletzten allerdings initial zunächst nur eine geschlossene Reposition mit grober Einrichtung und temporärer Fixateur-externe-Fixierung infrage. Die exakte anatomische offene Rekonstruktion erfolgt sekundär in Abhängigkeit vom Allgemeinzustand des Patienten. Bei offenen Frakturen erfolgt in dieser Situation die Erstversorgung mittels Débridement, vorübergehender Kunsthautdeckung und Fixateurstabilisierung [16-18]. Ausgedehntere Weichteilschäden erfordern die sekundäre plastische Deckung [5].

Aufgrund der kräftigen Bandverbindungen können Luxationsfrakturen der Chopart- und Lisfranc-Gelenklinie nur durch hohe lokale Gewalteinwirkung entste-
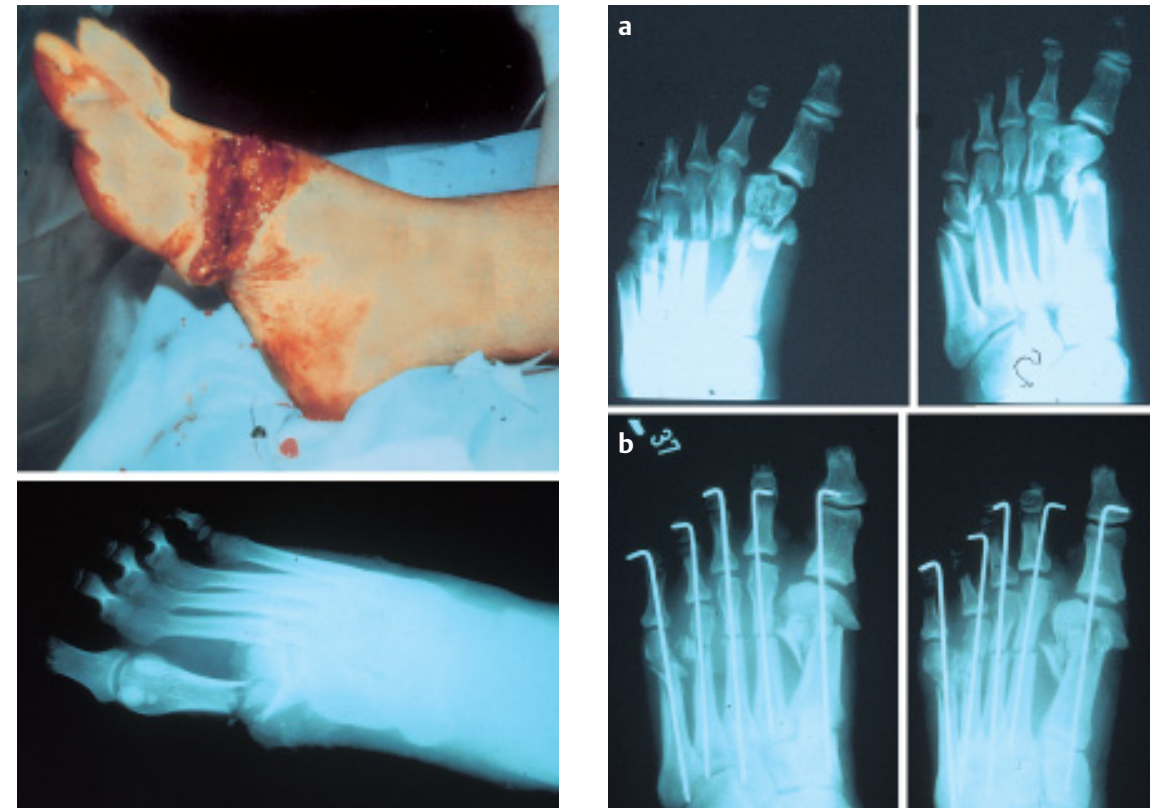

Abb.11 Rasenmäherverletzung mit III ${ }^{\circ}$ offener MT-I-Fraktur.

hen. Sie finden sich daher häufig im Rahmen einer Kettenverletzung der unteren Extremität bei Polytraumatisierten und weisen meist einen schweren Weichteilschaden auf. Bei schwerverletzten Patienten werden diese Verletzungen häufig übersehen. Wichtig ist daher vor allem das „Daran denken“ bei entsprechender Traumaanamnese. Reine und vollständige Luxationen in der Chopart- und Lisfranc-Linie sind selten. Meist sind sie kombiniert mit großen knöchernen Bandausrissen oder Fersenbeinbrüchen $[8,11]$. Sofern es der Allgemeinzustand des Patienten erlaubt, sollte daher im Rahmen der Primärdiagnostik eine exakte radiologische Diagnostik durch Standardprojektionen in 3 Ebenen (exakt seitlich, $45^{\circ}$ Schrägaufnahme, dorsoplantar mit $20^{\circ}$ dokranialer Kippung) erfolgen [10].

Luxationsfrakturen der Fußwurzel stellen die häufigste Ursache für ein Fußkompartment dar.

Luxationsfrakturen der beiden Gelenklinien stellen die häufigsten Ursachen für ein Fußkompartment dar und erfordern im Zweifelsfall die notfallmäßige Fasziotomie. Wenn keine Symptome eines Kompartmentsyndroms bestehen, sollte die Reposition geschlossen erreicht und mit Kirschner-Drähten gesichert werden, um eine zusätzliche Traumatisierung der
Abb.12 Frakturen aller 5 Mittelfußknochen nach Überrolltrauma (a). Operatives „Overtreatment" durch Stabilisierung sämtlicher Mittelfußknochen mit Kirschner-Drähten (b).

Weichteile in Grenzen zu halten. Sofern eine anatomische Reposition geschlossen nicht erreicht werden kann, bei intraartikulär liegenden Spongiosafragmenten sowie dislozierten Gelenkfragmenten sollte offen vorgegangen werden:

- Fasziotomie

- exaktes Gelenk-Debridement

- Refixation größerer gelenktragender Anteile mit Minischrauben und/oder Bohrdrähten

- Refixation von Bandverbindungen

Zusätzlich empfiehlt sich bei schwerem Weichteilschaden zur besseren Wundkontrolle sowie zur Spitzfußprophylaxe eine Sprunggelenktransfixation mit dem Fixateur externe $[8,11,16-18]$.

Metatarsalefrakturen finden sich häufig beim Komplextrauma mit schwerem Weichteilschaden infolge direkter Traumatisierung durch Überrollen bzw. Kontusion durch eine schwere Last, im Sommer häufiger auch durch Rasenmäherverletzungen (Abb.11). Im Zuge der Notfallversorgung muss die Dekompression eines Kompartmentsyndroms sowie die Versorgung offener Frakturen erfolgen. Häufig ist aufgrund des Weichteilschadens ein mehrzeitiges Therapiekonzept notwendig. Meist handelt es sich um basisnahe Frakturen. Das Prinzip besteht hier in der Stabilisierung der Hauptbelastungsträger des 1 . und 5. Metatarsale, die 
aufgrund des Weichteilschadens am besten minimal-invasiv durch Kirschner-Drähte erfolgen sollte. Eine zusätzliche ausgedehnte iatrogene Traumatisierung durch operative Stabilisierung aller 5 Mittelfußknochen ist für die Wiederherstellung eines belastbaren Fußskeletts nicht erforderlich und sollte daher unterbleiben (Abb.12). Je nach Weichteilsituation empfiehlt sich nach der operativen Fraktur- und Weichteilbehandlung die vorübergehende tibio-arsale Transfixation oder Immobilisierung in einer USGipsschiene. Für die Nachbehandlung sind häufig die Weichteilverhältnisse der limitierende Faktor. Die knöcherne Situation erfordert in den meisten Fällen eine Teilbelastung über 4-6 Wochen im Unterschenkelgehgips oder Gipsschuh. Die Drähte können nach 6 Wochen wieder entfernt werden [7,].

\section{Schlussfolgerung}

- Für die Behandlungsstrategie des komplexen Fußtraumas muss zunächst zwischen der Monoverletzung und der Begleitverletzung beim Polytraumatisierten unterschieden werden.

a Beim schwerstverletzten Patienten ist die primäre Teilamputation gerechtfertigt sobald absehbar ist, dass durch aufwendige Erhaltungsversuche der Patient vital gefährdet wird.

- Notfallmäßig versorgt werden müssen auch beim Schwerverletzten Kompartmentsyndrome und offene Frakturen. Bei ausgedehnter Weichteiltraumatisierung hat sich bereits initial die Sprunggelenkstransfixation mittels Fixateur externe sowie die minimal-in- vasive Beseitigung grober Fehlstellungen und Luxationen des Fußskeletts durch Kirschner-Drähte bewährt.

- Das wahre Ausmaß des Weichteilschadens kann meist erst im Rahmen eines second-look bestimmt werden, welcher beim Erhaltungsversuch routinemäßig innerhalb von $48 \mathrm{~h}$ geplant werden sollte.

- Zentrale Talusluxationsfrakturen sollen beim Monoverletzten sofort, beim Polytraumatisierten nach den lebenserhaltenden Eingriffen mittels offener Reposition und Schraubenosteosynthese versorgt werden. Kalkaneusund Mittelfußfrakturen können mit aufgeschobener Dringlichkeit noch bis zu 10 Tagen nach dem Trauma versorgt werden.

\section{Literatur}

${ }^{1}$ Berwarth H, Seif El Nasr M, Kuner EH. Grundzüge der Behandlung bei Frakturen mit offenem und geschlossenem Weichteilschaden. OP-Journal 1996; 12: 11 -15

${ }^{2}$ Bettag C, Böhm HJ, Hierholzer G. Hautzugverfahren zum sekundären Wundverschluß. Op-Journal 1996; 12: 65-68

${ }^{3}$ Echtermeyer V. Kompartmentsyndrom des Fußes. Orthopäde 1991; 20: 76-79

${ }^{4}$ Johansen K, Daines M, Howey T, Helfet D, Hansen ST. Objective criteria accurately predict amputation following lower extremity trauma. J Trauma 1990; 30: 568-572

${ }^{5}$ Maurer F, Müller JE, Weller S. Die Sanierung von Haut-Weichteil-Defekten bei Frakturen durch Lappenplastiken. OP-Journal 1996; 12: $58-63$

${ }^{6}$ Probst J. Primäre und sekundäre Amputationstechniken im Fußbereich. Unfallchirurg 1989; 92: 155-158

${ }^{7}$ Randt Th, Zwipp H. Indikation und Technik der Osteosynthese am Fuß. OP-Journal 1997; 13: $192-198$
8 Randt T, Dahlen C, Schikore H, Zwipp H. Luxationsfrakturen im Mittelfußbereich - Verletzungen des Chopart- und Lisfranc-Gelenkes. Zentralbl Chir 1998; 123: 1257-1266

${ }^{9}$ Randt T, Dahlen C, Schikore H, Zwipp H. Verletzungen der Fußwurzel und des Mittelfußes. Unfallchirurg 1998; 101: 935-949

${ }^{10}$ Reichelt S, Zwipp H, Prokop M. Röntgendiagnostik des Fußes. Unfallchirurg 1989; 92 : 103-109

${ }^{11}$ Suren EG, Zwipp H. Luxationsfrakturen im Chopart- und Lisfranc-Gelenk. Unfallchirurg 1991; 92: 130-139

${ }^{12}$ Swoboda B, Scola E, Zwipp H. Operative Behandlung und Spätergebnisse des Fußkompartmentsyndroms. Unfallchirurg 1991; 94 : $262-266$

13 Thermann $\mathrm{H}$, Hüfner T, Schratt HE, Albrecht $\mathrm{K}$, Tscherne H. Therapie intraartikulärer Fersenbeinfrakturen bei Erwachsenen. Unfallchirurg 1999; 102: 152-166

${ }^{14}$ Urban M, Holz U. Die besondere Bedeutung der Décollementverletzung. OP-Journal 1996; 12: 16-21

${ }^{15}$ Voigt C, Rahmanzadeh R. der frische Hautund Weichteildefekt bei Frakturen. OP-Journal 1996; 12: $43-48$

${ }^{16}$ Zwipp H, Tscherne H, Berger A. Rekonstruktive Fußchirurgie nach Komplextraumen des Fußes. Unfallchirurg 1989; 92: 140-154

${ }^{17}$ Zwipp H. Chirurgie des Fußes. Springer Verlag Wien 1994

${ }^{18}$ Zwipp H, Dahlen C, Randt T, Gavlik JM. Komplextrauma des Fußes. Orthopäde 1997; 26 : $1046-1056$

Dr. med. Thomas Ambacher

Assistenzarzt

Dr. med. Bernd Wittner

Oberarzt

Prof. Dr. med. Ulrich Holz

Ärztlicher Direktor

Unfallchirurgische Klinik

Katharinenhospital Stuttgart

Kriegsbergstr. 60

70174 Stuttgart 